\title{
1 Integrating Pharmacies into Public Health Program Planning for 2 Pandemic Influenza Vaccine Response
}

3 Authors: Thomas J Fitzgerald, MPH, Yoonjae Kang, MPH, Carolyn B Bridges, MD, Todd Talbert, MA, Sara J Vagi,

$4 \quad$ PhD, Brock Lamont, MPA, and Samuel B Graitcer, MD

5 About the Authors:

6 Thomas Fitzgerald is an Oak Ridge Institute for Science and Education (ORISE) Fellow in the Immunization

7 Services Division, National Center for Immunization and Respiratory Diseases, Centers for Disease Control and

8 Prevention, Atlanta, GA; Yoonjae Kang, Carolyn Bridges, Brock Lamont, and Samuel Graitcer are with the 9 Immunization Services Division, National Center for Immunization and Respiratory Diseases, Centers for 10 Disease Control and Prevention, Atlanta, GA; Todd Talbert and Sara Vagi are with the Division of State and 11 Local Readiness, Office of Public Health Preparedness and Response, Centers for Disease Control and

12 Prevention, Atlanta, GA.

Corresponding Author: Thomas Fitzgerald

Email:ymi8@cdc.gov

Phone: 404-639-3108

Disclaimer:

The findings and conclusions in this paper are those of the authors and do not necessarily represent the views of the Centers for Disease Control and Prevention 
Acknowledgements:

34 This project was supported in part by an appointment to the Research Participation Program at the 35 Immunization Services Division, National Center for Immunization and Respiratory Diseases, U.S. Centers for

36 Disease Control and Prevention, administered by the Oak Ridge Institute for Science and Education through an

37 interagency agreement between the U.S. Department of Energy and Centers for Disease Control and

38 Prevention. The authors would like to thank Suchita Patel, DO, MPH, and Tara Vogt, PhD, MPH, for their

39 editorial assistance in preparing this manuscript. 


\section{Abstract}

Background

During an influenza pandemic, to achieve early and rapid vaccination coverage and maximize the benefit of an immunization campaign, partnerships between public health agencies and vaccine providers are essential. Immunizing pharmacists represent an important group for expanding access to pandemic vaccination. However, little is known about nationwide coordination between public health programs and pharmacies for pandemic vaccine response planning.

\section{Methods}

To assess relationships and planning activities between public health programs and pharmacies, we analyzed data from Centers for Disease Control and Prevention assessments of jurisdictions that received immunization and emergency preparedness funding from 2012-2015.

Results

Forty-seven (88.7\%) of 53 jurisdictions reported including pharmacies in pandemic vaccine distribution plans, $24(45.3 \%)$ had processes to recruit pharmacists to vaccinate, and $16(30.8 \%)$ of 52 established formal relationships with pharmacies. Most jurisdictions plan to allocate less than $10 \%$ of pandemic vaccine supply to pharmacies.

\section{Discussion}

While most jurisdictions plan to include pharmacies as pandemic vaccine providers, work is needed to establish formalized agreements between public health departments and pharmacies to improve pandemic preparedness coordination and ensure that vaccinating pharmacists are fully utilized during a pandemic. 


\section{Introduction}

An influenza pandemic with high morbidity and mortality is a serious threat to the US public, especially given the persistent detection of highly pathogenic influenza A strains in humans and the recent detection of novel avian influenza $A$ virus infections in US domestic and wild bird populations ${ }^{1-3}$. If a severe influenza pandemic emerges, a well-coordinated vaccination campaign using a safe and effective pandemic influenza vaccine would need to be quickly implemented to reduce morbidity and mortality ${ }^{4}$. One pandemic vaccination campaign strategy is for public health programs to use points of dispensing (PODs) or mass vaccination clinics, but these may be inadequate to vaccinate necessary numbers of people over an extended vaccination campaign, as personnel and other resources may become exhausted. Healthcare providers in office or clinic settings may become too overwhelmed treating sick patients to focus heavily on vaccination efforts. Additional strategies should be considered to rapidly vaccinate the public during a severe influenza pandemic.

Leveraging pharmacists as pandemic influenza vaccine providers is an increasingly popular strategy as pharmacists are trained to vaccinate ${ }^{5}$. Pharmacists' authority to vaccinate, particularly against influenza, has expanded throughout the US and, as a result, pharmacists are able to vaccinate in some way in every state, District of Columbia (D.C.), and Puerto Rico, and are increasingly familiar with vaccine management and delivery $^{6}$. In fact, one in four adults who receives a seasonal influenza vaccine does so in a pharmacy or retail setting $^{7}$. Furthermore, in the US, there are over 67,000 community pharmacies, 297,000 vaccinating pharmacists, and $86 \%$ of the population lives within five miles of a pharmacy ${ }^{8-10}$.

For pharmacies to be ready to provide vaccinations during a severe pandemic, it is important for them to understand planning and logistical differences between seasonal and pandemic influenza vaccination. During typical influenza seasons, vaccine providers purchase vaccine from wholesalers, distributors, and manufacturers. During an influenza pandemic, as occurred during the 2009 influenza A (H1N1) pandemic, the Federal government will likely procure all pandemic vaccine directly from manufacturers, and each state public health department will be provided a pro-rata allocation ${ }^{11}$. Healthcare providers who wish to provide pandemic influenza vaccinations, including pharmacists, must coordinate and enroll with their jurisdictions' public health program, rather than with their typical vendors, to order and receive pandemic vaccine ${ }^{11}$. To determine each provider's allocation, public health departments need providers' physical shipping addresses and information such as patient population sizes and demographics, as well as vaccine storage and handling capacities. 
Given these important differences between seasonal influenza vaccination and planning for vaccination delivery during a pandemic, it is essential for public health programs and vaccine providers, including pharmacies, to coordinate these logistics in advance to ensure that the population is rapidly vaccinated against a pandemic. Initially, public health programs should identify and maintain up-to-date points of contacts (POCs) at their pharmacy partners and engage them in discussions to improve routine immunization delivery as well as pandemic preparedness. Formal agreements, such as memoranda of understanding (MOUs), may be important planning documents to then clarify coordination in pandemic vaccine program planning in order to fully leverage pharmacies' strengths. State public health immunization programs are ideally situated to develop these agreements with pharmacies, as they will likely receive Federally purchased vaccine and maintain oversight of its allocation, distribution, and administration throughout their jurisdictions. However, little is known about the type of coordination between state and city public health programs and pharmacies in pandemic influenza vaccine response planning.

We assessed existing relationships and planning activities of public health programs with pharmacies by analyzing data collected by the Centers for Disease Control and Prevention (CDC) from 2012 - 2015 from state and city jurisdictions that receive public health emergency preparedness and immunization funding ${ }^{12,13}$.

\section{Methods:}

CDC monitors public health program activities through a number of mechanisms, including the Annual Report of immunization program activities (hereinafter Annual Report), as well as ad hoc evaluations conducted in preparation for and response to influenza outbreaks and potential pandemics ${ }^{12}$. During the 2013 CDC response to the initial identification of influenza $A(H 7 N 9)$ among humans in China, $C D C^{\prime}$ s Immunization Services Division conducted a survey, the 2013 H7N9 Vaccination Response Planning and Needs Assessment (hereinafter H7N9 Needs Assessment) among 65 state and city jurisdictions that receive preparedness or immunization funding ${ }^{12,13}$. In 2015, CDC's Division of State and Local Readiness conducted a follow-up survey, the Pandemic Influenza Readiness Assessment (PIRA), which included a section on vaccination planning, among 62 jurisdictions that receive Public Health Emergency Preparedness program funding ${ }^{13}$.

We conducted a descriptive analysis of pandemic vaccine preparedness and response data from Annual Reports in years 2012-2014 (the most recent years available), the 2013 H7N9 Needs Assessment, and the 2015 PIRA to assess integration of pharmacies and pharmacists into public health pandemic vaccine response planning. We used absolute counts and proportions for all 50 states, D.C., New York City, and Chicago, for a 
total of 53 jurisdictions that are awarded funding for both immunization and preparedness programs ${ }^{12,13}$. Eight territory and freely associated states' public health and pharmacy infrastructures vary greatly from those presented here, and were excluded from the analysis. In some cases, respondents wrote information in the comments section which did not correspond to their selected answer, or would not select an answer and write in comments. Here, authors coded jurisdictions' free text responses to the appropriate answer. When reported proportions did not total $100 \%$, we recalculated responses by dividing each individual response by the reported total so each jurisdiction's responses totaled $100 \%$. We performed analyses using Microsoft Excel and SAS 9.3. Pharmacies are defined in this analysis as independent and chain community pharmacies (including those located in retailers), excluding hospitals and compounding pharmacies.

Results were grouped into 3 main domains: (1) engaging, recruiting, and enrolling pharmacists as pandemic vaccine providers; (2) vaccine allocation and distribution planning; and (3) policy, formal agreements, and memoranda of understanding.

\section{Results}

\section{Engaging, Recruiting, and Enrolling Pharmacists as Pandemic Vaccine Providers}

In the 2014 Annual Report, 20 (37.7\%) of 53 jurisdictions had identified POCs for all pharmacy partners (including state or local pharmacy boards and associations and independent and chain community pharmacies) in their jurisdictions, 18 (34.0\%) had identified POCs for more than half, but not all pharmacy partners, 12 (22.6\%) had identified POCs for less than half, and three (5.7\%) had no POCs for pharmacy partners in their jurisdictions (Table 1). In the H7N9 Needs Assessment, 43 (81.1\%) of 53 jurisdictions reported that they maintained a list of healthcare providers and facilities in their jurisdiction that could potentially be recruited as pandemic vaccine providers; of those, 40 (93.0\%) included pharmacists. Twenty-four (45.3\%) jurisdictions reported that they had formal processes for recruiting pharmacists (Table 1).

In the 2013 H7N9 Needs Assessment, 40 (75.5\%) of 53 jurisdictions reported that registering and enrolling healthcare providers and facilities (such as pharmacists and pharmacies) to become pandemic influenza vaccine providers was a state health department activity, while $11(20.8 \%)$ indicated that it was a joint activity between state and local health departments (LHDs). Two (3.8\%) jurisdictions reported that LHDs are responsible (Table 1). Forty-five (88.2\%) of 51 responding jurisdictions reported in the 2015 PIRA that this was the responsibility of the city or state awardee health department, and 6 (11.8\%) reported that LHDs were responsible for this activity (Table 1). There was no response option for this being a joint activity on the 2015 
PIRA. Of the 11 programs reporting joint responsibility in 2013, nine reported that this was a state responsibility in the 2015 PIRA, and two reported that it was a local responsibility. Three programs that reported that this was a state responsibility in 2013 then reported that it was a local responsibility in 2015.

\section{Vaccine Allocation and Distribution Planning}

In the 2012 Annual Report, 47 (88.7\%) of 53 jurisdictions reported including pharmacies as pandemic vaccine providers in their vaccine distribution plans. Thirty-one (58.5\%) reported including pharmacies during early phases of their pandemic vaccine campaign compared to $16(30.2 \%)$ that include them only during late phases of their campaign. Six (11.3\%) indicated that pharmacies were not included in pandemic vaccine response plans at all. In the 2013 H7N9 Needs Assessment, 47 (88.7\%) of 53 jurisdictions reported that they would request pandemic vaccine be shipped directly to pharmacy locations using the CDC contracted distributor rather than allow pharmacies to use their own vaccine management and distribution infrastructure. Eight (16.0\%) of 50 jurisdictions reported in the 2014 Annual Report that they would allow pharmacy companies to allocate and distribute pandemic vaccine to individual stores using their existing systems rather than have the public health program manage their store allocations, while $13(26.0 \%)$ indicated that this would depend on supply and availability (Table 2).

Forty jurisdictions indicated in the 2013 H7N9 Needs Assessment that they had a list of potential pandemic influenza vaccine providers which included pharmacies (Table 1); of which, $12(30.0 \%)$ reported having information on the numbers of patients those pharmacies served, 20 (50.0\%) had information on the types of patient populations they served, and $14(35.0 \%)$ had information on their vaccine storage and handling capacities (Table 2). Two (5.0\%) jurisdictions indicated that they had pharmacists on their list of potential pandemic vaccine providers, but would not request to have vaccine shipped directly from CDC's contracted distributor to these providers during a pandemic. Four (10.0\%) reported having pharmacists as potential pandemic vaccine providers, but did not know if they would request shipments by CDC's contracted distributor to them during a pandemic. Two (5.0\%) did not report having pharmacists on their lists of potential vaccine providers, but reported that they would request that vaccine be shipped to pharmacies during a pandemic, and another (2.5\%) did not know if pharmacists were on their list of potential pandemic vaccine providers, but would request that vaccine be shipped to them.

Thirty-two (61.5\%) of 52 responding jurisdictions in the 2015 PIRA reported that more than $20 \%$ of their jurisdiction's vaccine allocation would be dispensed at PODs (Table 2). When asked to estimate the proportion of their jurisdiction's weekly vaccine allocation that they plan to allocate to different provider types, 29 
(60.4\%) of 48 respondents intended to provide $20 \%$ or more of their jurisdiction's weekly pandemic vaccine allocation to mass vaccination clinics or PODs (Table 3). Thirty-four (70.8\%) jurisdictions reported that they planned to send less than $5 \%$ of their weekly vaccine supply to retail-based clinics, 18 (37.5\%) planned to send less than $5 \%$ to large pharmacies, and $32(66.7 \%)$ planned to send less than $5 \%$ to local pharmacies. One (2.1\%) jurisdiction reported planning to send $20 \%$ of its vaccine to retail-based clinics, and 8 (16.7\%) reported plans to send $20 \%$ or more to large pharmacies. No jurisdictions reported planning to send $20 \%$ or more of its weekly vaccine supply to local pharmacies.

\section{Policy, Formal Agreements, and Memoranda of Understanding}

Seven (13.5\%) of 52 responding jurisdictions reported in the 2014 Annual Report that pharmacists would be directed to vaccinate all patients regardless of a patient's insurance status or ability to pay for vaccine administration. Three (5.8\%) programs would instruct pharmacists to refer these patients to health departments for vaccination, and 32 (61.5\%) had not clarified this issue.

Seventeen (35.4\%) of 48 responding jurisdictions indicated in the 2013 Annual Report that they had established or provided support for LHDs to establish MOUs or formalized preparedness agreements with pharmacies to distribute and administer vaccine during a pandemic (Table 4). Five (9.6\%) of 52 responding jurisdictions in the 2014 Annual Report reported establishing pandemic influenza vaccine MOUs for all pharmacies in their jurisdictions, five (9.6\%) established them for over half, but not all, pharmacy groups, while six (11.5\%) established such agreements for less than half of pharmacy groups. Thirty-three (63.5\%) jurisdictions had no agreement in place, and three (5.7\%) did not know (Table 4). Nine jurisdictions who did not respond or did not have pharmacy MOUs in 2013 reported having one with any pharmacy in 2014, while eight that had indicated having or supporting an MOU with pharmacies in 2013 reported that they did not have one in 2014.

\section{Discussion:}

CDC supports the role of pharmacies in pandemic influenza vaccine planning and response ${ }^{14}$. Our analysis of self-reported data from public health programs collected by CDC from 2012-2015 suggests that most programs view pharmacies and pharmacists as valuable partners in pandemic vaccination activities and do plan to include pharmacists as vaccinators during the next pandemic, but significant planning gaps remain. These include incomplete identification of POCs, inconsistent processes for recruiting pharmacists as pandemic vaccinators, lack of clarity on the responsibility for outreach to and enrollment of pharmacists as 
pandemic vaccinators, inconsistencies in jurisdictions' pandemic vaccine allocation and distribution plans to pharmacies, and limited information on pharmacy patient populations and storage and handling capacity needed to make rapid vaccine allocation decisions. In addition, issues such as vaccinating uninsured patients in pharmacies have not been clarified, and few jurisdictions have formal agreements or MOUs in place to improve pandemic vaccine response logistics. Perhaps the biggest pandemic vaccine program planning gap is that most public health programs appear to be underestimating the value and potential vaccination capacity pharmacies and pharmacists might have in rapidly vaccinating the public during a pandemic. For example, most programs reported that they would allocate only a small portion of their pandemic influenza vaccine supply to pharmacies despite the fact that pharmacies have demonstrated their ability to vaccinate much larger portions of the public for seasonal influenza, now vaccinating roughly $25 \%$ of adults who receive seasonal influenza vaccine ${ }^{7}$.

While pharmacists provided vaccinations to the public during the 2009 influenza $A(H 1 N 1)$ pandemic, that vaccine did not become widely available to pharmacies until December 2009, after the disease had peaked in October 2009 and public demand for vaccination had waned ${ }^{15}$. Biggerstaff, et. al. found that in a modeled vaccination campaign during a severe pandemic, assuming vaccination at a constant rate, the timing of the start of vaccination was the greatest factor in reducing morbidity and mortality, and vaccination beginning just prior to or at disease peak would have little impact in mitigating deaths and hospitalizations associated with the pandemic ${ }^{16}$. Schwerzmann and colleagues found that including pharmacies in a pandemic influenza vaccine campaign, despite high participation from other vaccinators, can significantly reduce the time necessary to vaccinate $80 \%$ of US adults with a single dose, from 18 to 11 weeks, assuming high demand for vaccination and sufficient supply ${ }^{17}$. However, in our analysis we found that some public health programs plan to include pharmacists as vaccinators later in a pandemic vaccine response, rather than earlier.

While Stergachis, et. al. found that $94 \%$ of pharmacists would be willing to work during an influenza pandemic, Seib and colleagues found that 2009 H1N1 vaccine providers who had participated in emergency training or response were more willing to respond to a public health emergency ${ }^{18,19}$. In another study by Pederson, et. al., the only factor influencing pharmacists' opinions regarding their level of involvement in emergency preparedness and response was previous participation in local or state emergency preparedness and response activities $^{20}$. As part of the disaster health workforce with specialized training and skills, it is vital for pharmacists to be familiar with public health core competencies and considered in planning stages ${ }^{22}$. 
However, a 2012 poll showed that $68 \%$ of pharmacists working in community settings had no contact with health department staff in the previous year ${ }^{21}$.

Our findings are subject to several limitations. Survey respondents were predominantly immunization and emergency preparedness programs at the state level, but many states have local or regional health departments which maintain jurisdiction over public health activities. No data were collected from pharmacies, pharmacists, or pharmacy boards. The data presented here are self-reported, come from a variety of sources, and might have been interpreted and answered differently, depending on the person or program responsible for completing any particular assessment, all of which may limit comparability. On the Annual Report, respondents are able to skip questions, and data are not verified. The 2013 H7N9 Readiness Assessment was distributed with relatively little time to complete it, while also requiring coordination between preparedness and immunization staff. The wording of questions on the same assessment differ from year to year, making temporal trends difficult to assess.

It is crucial that roles and responsibilities between public health programs and pharmacies are outlined in advance to ensure clarity and consistency in a pandemic vaccine response, but our analysis suggests this may not be occurring regularly or with a consistent approach. One way to ensure a more consistent approach is by formalized pre-pandemic agreements, such as MOUs, between public health departments and pharmacies. The Association of State and Territorial Health Officials (ASTHO), in collaboration with representatives from $\mathrm{CDC}$, the pharmacy industry, and immunization programs, developed a template MOU to serve as a model to outline roles and responsibilities between public health programs and pharmacies in preparing for a pandemic influenza vaccine response. The template MOU outlines best practices for recruiting and training vaccine providers, vaccine allocation and distribution to pharmacies, vaccine administration data and tracking, and pandemic vaccine cost and payment issues ${ }^{23}$. The template provides a standardized approach for coordination between state public health programs and pharmacies operating in many states, but allows for flexibility in implementation to meet unique jurisdictions' needs. As of September 2016, the template is being piloted by three states. CDC and ASTHO are working with their immunization and preparedness partners to disseminate and raise awareness of this preparedness tool ${ }^{23}$.

Our analysis underscores gaps in efforts to improve planning efforts between public health programs and pharmacies, a partnership which may be vital to achieving sufficient vaccination coverage during a severe pandemic. While state public health programs appear willing to work with pharmacies to improve pandemic vaccine preparedness and response, it is important to develop strategies allowing for early and ongoing 
321 involvement in planning and response activities in order to minimize vulnerability to and optimize their

322 jurisdictions' preparedness for a pandemic. Improved and formalized relationships between public health programs and pharmacies may allow for the widespread use of pharmacists as vaccinators during the next pandemic, greatly increasing vaccination coverage and reducing morbidity and mortality associated with the pandemic. 


\section{Appendix A: Full-text Awardee Survey Questions}

\section{Engaging, recruiting, and enrolling vaccine providers:}

\section{H7N9 Needs Assessment}

Who would manage the process of enrolling and registering health care providers and facilities interested in serving as pandemic vaccine providers? (check one)

State health department

Local health departments

Regional health departments

Joint activity between state health department and local/regional health departments

Other

Does your jurisdiction have a list of health care providers and facilities that have practice locations in your jurisdiction that could potentially be recruited as H7N9 vaccine providers? [Note: this would represent the overall number (i.e., denominator) of providers and facilities in your jurisdiction, not just those that have expressed interest in becoming pandemic influenza vaccine providers or those who were enrolled and registered as H1N1 vaccine providers]

Yes

No

No, because maintaining such lists is the responsibility of our local health departments

If YES to having a list of potential vaccine providers and facilities in your jurisdiction: Please indicate whether the following types of providers and facilities are included in your list.

Pharmacies

Yes

No

Don't Know

Does your jurisdiction have a formal or written process for recruiting the following types of healthcare providers and facilities to administer a pandemic vaccine?

Pharmacists

Yes

No

Don't Know

\section{PIRA}

Which entity within your jurisdiction would have primary responsibility for activities related to registering and enrolling healthcare providers and facilities to become pandemic influenza vaccine providers?

Awardee health department

Local health department(s)

Regional health department(s)

Other 


\section{Annual Report}

Have you identified points of contact for pharmacy partners in your jurisdiction, such as with state or local pharmacy boards, pharmacy associations, national pharmacy chains operating in your jurisdiction, retails stores that include immunizing pharmacists, and independent pharmacies?

Yes, we have identified points of contact for all pharmacy partners in our jurisdiction

We have identified points of contact for more than half, but not all, pharmacy partners in our jurisdiction

We have identified points of contact for less than half of the pharmacy partners in our jurisdiction

We have not identified any points of contact for any pharmacy partners in our jurisdiction

\section{Vaccine allocation and distribution planning;}

\section{Annual Report}

Do your current pandemic vaccine distribution plans include pharmacies and retail based clinics as vaccine providers:

Early in a pandemic response

Later in a pandemic response

Our immunization program does not have current plans for including pharmacies and retail based clinics as vaccine providers

\section{H7N9 Needs Assessment}

If a pandemic vaccination program were initiated, to which location(s) or types of providers would your jurisdiction request pandemic vaccine be shipped from CDC's centralized distribution contractor?

Pharmacies

Yes

No

Don't Know

If YES to having a list of potential vaccine providers and facilities in your jurisdiction: Which of the following types of information do you have about most or all of the providers and facilities on your list?

Estimated number of patients served

Patient population served (e.g. pregnant women, adults plus children, children only, adults only)

Occupational groups served (e.g. first responders, healthcare workers, other critical infrastructure)

Vaccine storage and handling capacity

Physical location address

Phone numbers

Email addresses

Fax numbers

\section{Annual Report}


If you have a formal relationship or agreement with pharmacies to provide pandemic vaccinations during a response, we would like to learn how you will maximize the current distribution capacity of these chain pharmacies or retail stores during a pandemic. Does your jurisdiction's plan allow pharmacies to allocate and distribute to individual stores using their existing distribution and management infrastructure

Yes

We have not discussed this

It depends on vaccine supply and availability

Other

If other, please describe

\section{$\underline{2015 \text { PIRA }}$}

Does your jurisdiction plan to provide more than $20 \%$ of your vaccine allocation through PODs?

Yes

No

Based on your pandemic plan and knowledge about your jurisdiction, please estimate the proportion of your jurisdiction's weekly vaccine allocation that you plan to allocate to each of the following type of provider group or venue when there is ample vaccine supply.

Closed Points of dispensing (PODs)

Open Points of dispensing (PODs)

School-located vaccination Clinics (SLVs)

Providers already enrolled as Vaccines for Children (VFC) providers

Non-VFC pediatric providers

Non-VFC adult providers

Community Vaccinators such as visiting nurses associations

Large retail-based or grocery stores

Large national or regional chain pharmacies

Independent, local pharmacies

Local health departments (LHDs)

Other

\section{Policy, formal agreements, and memoranda of understanding.}

\section{Annual Report}

Does your program provide support to regional or local health department programs to develop Memorandums of Agreement with groups that would provide vaccination services to critical infrastructure personnel that may be prioritized for vaccine in a pandemic?

Yes

No

Don't Know

Comment

If yes, please briefly describe your program support in this effort:

If no or don't know, please provide a reason: 
Does your jurisdiction, either directly through the immunization program or through the public health preparedness program, have or provide support to local or regional public health programs to establish formalized relationships or Memorandums of Agreement/ Understanding (MOA/U) with pharmacies to distribute and administer pandemic vaccine during a pandemic, outside of the general provider agreement?

Yes, we have such agreement in place for all pharmacy groups in our jurisdiction

Yes, we have such agreement in place for more than half, but not all, of pharmacy groups in our jurisdiction

Yes, we have such agreement in place for less than half of pharmacy groups in our jurisdiction We have no such agreements in place with pharmacies in our jurisdiction Don't Know

Though it is likely that all pandemic vaccines and constituent products would be provided by the Federal government at no charge during a pandemic, some providers may also bill patients for vaccine administration fees. We'd like to learn about what guidance pharmacies have been given regarding providing vaccination services to uninsured and underinsured groups during a future pandemic. Regarding uninsured and underinsured patients during a pandemic, pharmacists have been instructed to:

Refer these patients to the public health department for pandemic vaccination

Provide Pandemic vaccinations to all customers/patients regardless of insurance status or ability to pay

This topic has not yet been clarified

Other

If Other, please describe 
495 Conflicts of Interest:

496 The authors have no conflicts of interest to disclose

497

498

499 
References:

1. Holloway R, Rasmussen SA, Zaza S, Cox NJ, Jernigan DB. Updated preparedness and response framework for influenza pandemics. MMWR. Recommendations and reports : Morbidity and mortality weekly report. Recommendations and reports / Centers for Disease Control. 2014;63(Rr-06):1-18.

2. Centers for Disease Control and Prevention (CDC). How the Flu Virus Can Change: "Drift" and "Shift". 2014; http://www.cdc.gov/flu/about/viruses/change.htm. Accessed November 24, 2015.

3. Centers for Disease Control and Prevention (CDC). Avian Influenza Current Situation. 2015; http://www.cdc.gov/flu/avianflu/avian-flu-summary.htm. Accessed February 2, 2016.

4. World Health Organization (WHO). Questions and Answers on Pandemic Influenza Vaccine. Immunization, Vaccines and Biologicals 2007; http://www.who.int/immunization/newsroom/PI QAs/en/. Accessed April 11, 2016.

5. $\quad$ American Pharmacists Association (APhA). Pharmacy-Based Immunization Delivery. 2016; http://www.pharmacist.com/pharmacy-based-immunization-delivery. Accessed August 31, 2016.

6. American Pharmacists Association (APhA). Pharmacist Immunization Authority. 2013; http://www.pharmacist.com/sites/default/files/PharmacistlZAuthority.pdf. Accessed August 31, 2016.

7. Srivastav A, Williams WW, Santibanez TA, et al. National Early Season Flu Vaccination Coverage, United States, November 2015. FluVaxView 2015; http://www.cdc.gov/flu/fluvaxview/nifs-estimates-nov2015.htm. Accessed February 1, 2016, 2016.

8. National Association of Chain Drug Stores (NACDS) Economics Department. Chain Pharmacy Industry Profile. Internal Document2016.

9. $\quad$ SK\&A. National Pharmacy Market Summary: Market Insights Report. March 20162016.

10. Rothholz MC. Pearls of Wisdom: Instilling the Importance of Vaccinations - From Student to Practitioner. Paper presented at: National Adult and Influenza Immunization Summit; May 10, 2016, 2016; Atlanta, GA.

11. U.S. Department of Health and Human Services (HHS). HHS Pandemic Influenza Plan. In: Department of Health and Human Services, ed2005.

12. Centers for Disease Control and Prevention (CDC). Vaccines for Children Program (VFC). 2014; http://www.cdc.gov/vaccines/programs/vfc/index.html. Accessed December 1, 2015.

13. Centers for Disease Control and Prevention (CDC). Funding and Guidance for State and Local Health Departments. 2015; http://www.cdc.gov/phpr/coopagreement.htm. Accessed February1, 2016, 2016.

14. Schuchat A. Letter to Pharmacists. In: (CDC) CfDCaP, ed2015.

15. Koonin LM, Beauvais DR, Shimabukuro T, et al. CDC's 2009 H1N1 vaccine pharmacy initiative in the United States: implications for future public health and pharmacy collaborations for emergency response. Disaster medicine and public health preparedness. 2011;5(4):253-255.

16. Biggerstaff M, Reed C, Swerdlow DL, et al. Estimating the Potential Effects of a Vaccine Program Against an Emerging Influenza Pandemic-United States. Clinical Infectious Diseases. 2015;60(S1):10.

17. Schwerzmann J. Modeling Pandemic Influenza Vaccine Administration (Not Yet Published). Battelle Memorial Institute, Centers for Disease Control and Prevention; 2015.

18. Stergachis A, Garberson L, Lien O, D'Ambrosio L, Sangare L, Dold C. Health care workers' ability and willingness to report to work during public health emergencies. Disaster medicine and public health preparedness. 2011;5(4):300-308.

19. Seib K, Barnett DJ, Weiss PS, Omer SB. Vaccine-related standard of care and willingness to respond to public health emergencies: a cross-sectional survey of California vaccine providers. Vaccine. 2012;31(1):196-201.

20. Pedersen CA, Canaday BR, Ellis WM, et al. Pharmacists' opinions regarding level of involvement in emergency preparedness and response. Journal of the American Pharmacists Association : JAPhA. 2003;43(6):694-701.

21. Harvard Opinion Research Program. The voice of pharmacists: a poll about alternative methods for antiviral distribution during a pandemic influenza. 2012.

22. Walsh L, Subbarao I, Gebbie K, et al. Core competencies for disaster medicine and public health. Disaster medicine and public health preparedness. 2012;6(1):44-52.

23. Association of State and Territorial Health Officials (ASTHO). Memorandum of Understanding for the Coordination of A Pandemic Influenza Vaccination Campaign in Planning for and Responding to An Influenza 
550

551

Pandemic. http://www.astho.org/Infectious-Disease/Pandemic-Influenza/MOU-State-Pharmacy-PandemicInfluenza-Vaccination-Campaign/2015. 
Table 1. Public Health Program Plans for Recruiting, Enrolling, and Registering Pharmacists as Pandemic Influenza Vaccine Providers

\begin{tabular}{|c|c|c|}
\hline Assessment & No. (\%) & To \\
\hline $\begin{array}{l}\text { Primary responsibility for activities related to registering and enrol } \\
\text { become pandemic influenza vaccine providers, } 2013^{1}\end{array}$ & & \\
\hline State health department & $40(75.5 \%)$ & \\
\hline Local health departments $\ddagger$ & $2(3.8 \%)$ & \\
\hline Joint activity between state and local health departments & $11(20.8 \%)$ & \\
\hline
\end{tabular}

Primary responsibility for activities related to registering and enrolling healthcare providers and facilities to become pandemic influenza vaccine providers, $2015^{2}$

Awardee state or city health department

Local health departments ${ }^{\S}$

$45(88.2 \%) \quad 51$

$6(11.8 \%) \quad 51$

Identified points of contact for pharmacy partners in jurisdiction ${ }^{3}$

Yes, for all pharmacy partners

Yes, for more than half, but not all, pharmacy partners

Yes, for less than half of pharmacy partners

No

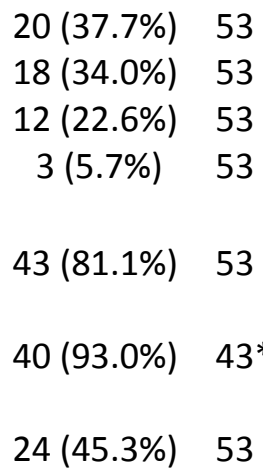

Formal process for recruiting pharmacists as pandemic vaccinators has been established ${ }^{1}$

$24(45.3 \%) \quad 53$

Data Sources: 1: 2013 H7N9 Needs Assessment: 2: 2015 Pandemic Influenza Readiness Assessment 3: 2014 Annual Report

*: Subset of above assessment

+: Responses do not always equal 53 due to missing responses from jurisdictions

¥: Local health departments include directly funded cities (Chicago, New York City, and Washington, D.C.)

$\S$ : Local health departments do not include directly funded cities 
Table 2 - Public Health Program Plans for Vaccine Allocation and Distribution of Pandemic Vaccine to Community Pharmacies

\begin{tabular}{|c|c|c|}
\hline Assessment & No. (\%) & Total Responses* \\
\hline \multicolumn{3}{|l|}{ Current pandemic vaccine distribution plans include pharmacists: ${ }^{1}$} \\
\hline Early in a response & $31(58.5 \%)$ & 53 \\
\hline Later in a response & $16(30.2 \%)$ & 53 \\
\hline No plans for including pharmacists & $6(11.2 \%)$ & 53 \\
\hline Program will request pandemic vaccine be shipped to pharmacies from CDC's contracted distributor ${ }^{2}$ & $47(88.7 \%)$ & 53 \\
\hline \multicolumn{3}{|l|}{ Pharmacies allowed to reallocate and distribute vaccine to individual stores using existing system: ${ }^{3}$} \\
\hline Yes & $8(16.0 \%)$ & 50 \\
\hline Depends on vaccine supply and availability & $13(26.0 \%)$ & 50 \\
\hline Not discussed & 14 (28.0\%) & 50 \\
\hline Other & $15(30.0 \%)$ & 50 \\
\hline \multicolumn{3}{|l|}{ Programs have information needed to make allocation and distribution decisions for pharmacies: ${ }^{2} \neq$} \\
\hline Physical location/address & $37(92.5 \%)$ & $40^{+}$ \\
\hline Email & $29(72.5 \%)$ & $40^{+}$ \\
\hline Number of patients served & $12(30.0 \%)$ & $40^{+}$ \\
\hline Type of patient population served & $20(50.0 \%)$ & $40^{+}$ \\
\hline Vaccine storage and handling capacity & $14(35.0 \%)$ & $40^{+}$ \\
\hline Plan to provide more than $20 \%$ of jurisdiction's vaccine allocation through points of dispensing ${ }^{4}$ & $32(61.5 \%)$ & 52 \\
\hline
\end{tabular}


Table 4 - Immunization Policy, Formalized Agreements, and Memoranda of Understanding between Public Health and Community Pharmacies

\begin{tabular}{|c|c|c|}
\hline Assessment & No. (\%) & Total Responses ${ }^{+}$ \\
\hline \multicolumn{3}{|c|}{ Regarding uninsured and underinsured patients during a pandemic, pharmacists have been instructed to: ${ }^{1}$} \\
\hline Provide pandemic vaccinations to all patients regardless of ability to pay & 7 (13.5\%) & 52 \\
\hline Refer these patients to the health department & $3(5.8 \%)$ & 52 \\
\hline Not clarified & $32(61.5 \%)$ & 52 \\
\hline Other & $10(19.2 \%)$ & 52 \\
\hline \multicolumn{3}{|c|}{$\begin{array}{l}\text { Have or provide support to local health departments to establish memoranda of understanding or } \\
\text { formalized agreements with community pharmacies in } 2013^{2}\end{array}$} \\
\hline Yes & $17(35.4 \%)$ & 48 \\
\hline No & $21(43.8 \%)$ & 48 \\
\hline Other & $10(20.8 \%)$ & 48 \\
\hline \multicolumn{3}{|c|}{$\begin{array}{l}\text { Have or provide support to local health departments to establish memoranda of understanding or } \\
\text { formalized agreements with community pharmacies in } 2014^{1}\end{array}$} \\
\hline Yes, for all pharmacy partners & $5(9.6 \%)$ & 52 \\
\hline Yes, for more than half, but not all, pharmacy partners & $5(9.6 \%)$ & 52 \\
\hline Yes, for less than half of pharmacy partners & $6(11.5 \%)$ & 52 \\
\hline No & $33(63.5 \%)$ & 52 \\
\hline Don't Know & $3(5.7 \%)$ & 52 \\
\hline
\end{tabular}

Data Sources: 1: 2014 Annual Report; 2: 2013 Annual Report

$*$ : Responses do not always equal 53 due to missing responses from jurisdictions 